\title{
ESTADO DEL ARTE SOBRE EL CAMBIO CLIMÁTICO Y LAS AGUAS SUBTERRÁNEAS. EJEMPLOS EN COLOMBIA
}

\author{
Silvana Bolaños Chavarría ${ }^{1}$, Teresita Betancur Vargas² \\ ${ }^{1}$ Ingeniera Ambiental, Universidad de Antioquia. Grupo de Investigación GIGA. Medellín-Colombia. \\ silvana.bolanos@udea.edu.co \\ 2Doctora en Ingeniería, Profesora Titular Universidad de Antioquia. Grupo de Investigación GIGA. Medellín- \\ Colombia. teresita.betancur@udea.edu.co
}

\begin{abstract}
RESUMEN
Si bien existen muchas alusiones en cuanto a los efectos del cambio climático en el ciclo hidrológico global, éstas se encuentran referidas generalmente a la temperatura atmosférica, la precipitación y al incremento del nivel del mar, y poco se habla de los efectos sobre el recurso hídrico subterráneo. A partir de un ejercicio de revisión bibliográfica que incluyó alrededor de 60 referencias de los últimos 10 años, se empieza a consolidar un marco de referencia al respecto, y se efectúa una primera aproximación a la evaluación de los efectos del cambio climático sobre tres sistemas hidrogeológicos en Colombia. Considerando los escenarios de cambio climático propuestos por el IDEAM, se practica un análisis conceptual de las posibles implicaciones sobre la recarga para los acuíferos del Valle de Aburrá, Urabá antioqueño y Cuenca del rio Ranchería, que conllevaría a una disminución de la descarga de aguas subterráneas y un impacto directo en ecosistemas dependientes.
\end{abstract}

Palabras clave: Acuíferos; disminución de la recarga; fase subterránea del ciclo-hidrológico

Recibido: 27 de Julio de 2017. Aceptado: 7 de Junio de 2018

Received: July 27th, $2017 . \quad$ Accepted: June 7th, 2018

\section{STATE OF THE ART ON CLIMATE CHANGE AND GROUNDWATER. EXAMPLES IN COLOMBIA}

\begin{abstract}
Although there are many allusions concerning the effects of climate change on the global hydrological cycle, these allusions are generally referred to atmospheric temperature, rainfall and rising sea levels, but the effects on groundwater resources are rarely discussed. From a bibliographic review exercise that included around 60 references from the last 10 years, a framework is being consolidated in this regard, and a first approximation is made to the evaluation of the effects of climate change on three hydrogeological systems in Colombia. Considering the climate change scenarios proposed by the IDEAM, a conceptual analysis of the possible implications on the recharge is practiced for the aquifers of the Aburrá Valley, Urabá antioqueño and Ranchería River Basin, which would lead to a decrease in groundwater discharge and a direct impact on dependent ecosystems.
\end{abstract}

Keywords: Aquifers; decrease in recharge; underground phase of hydrologic cycle

Cómo citar este artículo: S. Bolaños Chavarría, T. Betancur Vargas, "Estado del arte sobre el cambio climático y las aguas subterráneas. Ejemplos en Colombia" Revista Politécnica, Año 14, Vol 14, No 26, pp 52-64, 2018. https://doi.org/10.33571/rpolitec.v14n26a5 


\section{INTRODUCCIÓN}

A causa de la necesidad de contar con nuevas fuentes hídricas, cada vez se hace más evidente que las aguas subterráneas han sido, son y pueden llegar a ser la principal fuente de abastecimiento de agua para consumo humano en el mundo. Ellas constituyen la principal y más grande reserva de agua dulce líquida del planeta, 95\% descontando los casquetes polares y los hielos glaciares, una cifra bastante considerable comparada con el agua de los ríos que suma aproximadamente el 0,006\% del total [1]. Las aguas subterráneas son una fuente importante de agua potable para casi la mitad de la población mundial [2]; globalmente es la fuente de un tercio de toda el agua dulce extraída [3]; proporciona agua para consumo humano, agricultura, la industria y muchos ecosistemas dependientes. No cabe duda que el uso, la conservación, recuperación y en general, la gestión del agua subterránea actualmente debe tener en cuenta la preocupación de carácter global que cada vez más aumenta con el paso del tiempo: el Cambio Climático. Esta preocupación, en el tema hidrogeológico, se deriva de la posible reducción de las precipitaciones y el aumento de la evapotranspiración, las cuales afectarían la recarga $y$, posiblemente, aumentará las tasas de demanda de agua subterránea [4]-[6]. La importancia estratégica del agua subterránea para el abastecimiento y seguridad alimentaria, probablemente se intensificará bajo el cambio climático, en la medida en que más frecuentes e intensos sean los extremos climáticos (inundaciones y sequías) [7].

En comparación con las aguas superficiales, el nivel de atención prestado a las aguas subterráneas, especialmente en países en desarrollo es bastante escaso y limitado, lo que refleja un descuido general de su gestión, así como las incertidumbres sobre los posibles impactos del cambio climático [4], [7]-[11]. Aún no se conoce el grado en el que las captaciones de aguas subterráneas se han visto afectados por el cambio climático, así que tanto la detección de cambios en los sistemas hidrogeológicos como la atribución de estos al cambio climático, son raros y difíciles debido a la falta observaciones apropiadas y a la existencia de un número pequeño de estudios. Las incertidumbres en la recarga proyectada de acuíferos que se originan en los modelos hidrológicos aún no han sido exploradas. Hay sólo unos pocos estudios sobre los impactos en las aguas subterráneas debido a cambios de vegetación en respuesta al cambio climático y el aumento de $\mathrm{CO} 2$, y tampoco existen estudios sobre el impacto inducido por cambios en el uso del suelo en la recarga de acuíferos [12]. Además, los impactos directos del cambio climático en los procesos naturales de la recarga de acuíferos, descarga, almacenamiento, intrusión salina, reacciones biogeoquímicas y transporte de químicos, pueden ser aumentados por las actividades humanas [5].

Por ello, tanto por sus características como por el grado de desconocimiento, es crucial que se realicen cada vez más estudios alrededor de este bien invisible. En este artículo, se reúne y se sintetiza información de estudios alrededor del mundo sobre el impacto del cambio climático en las aguas subterráneas, con el propósito de realizar posteriormente un análisis conceptual en tres sistemas acuíferos de Colombia teniendo en cuenta los escenarios de cambio climático en el país. Sea cual sea el escenario futuro, se debe pensar en el agua subterránea como un recurso que juega un rol importante en la adaptación a la variabilidad hidrológica y al cambio climático [4] y que, por ende, es necesaria una gestión integral del recurso hídrico teniendo en cuenta los posibles impactos que sobre las aguas subterráneas podrían tener el cambio climático.

\section{CAMBIO CLIMÁTICO Y AGUAS SUBTERRÁNEAS}

Aunque la importancia de la relación entre las aguas subterráneas y el cambio climático no puede ser exagerada 0 menospreciada, y pese a no contar con indicaciones precisas, de no tener una gestión adecuada, las aguas subterráneas pueden resultar en uno de los elementos más afectados por un posible cambio climático dadas sus particularidades en el contexto del ciclo hidrológico [13]. Cualquier variación en el régimen y cantidad de la precipitación, junto con variaciones en la temperatura y evapotranspiración, pueden afectar la recarga, descarga y calidad de las aguas subterráneas [4], [14], [15].

Haciendo una revisión en la literatura en la que se incluye aproximadamente 60 referencias de los últimos 10 años, los posibles efectos del cambio climático en cada uno de los procesos que conforman la fase subterránea del ciclo hidrológico 
se pueden resumir en los aspectos mencionados en la Fig.1 [4], [6], [12], [14], [16].

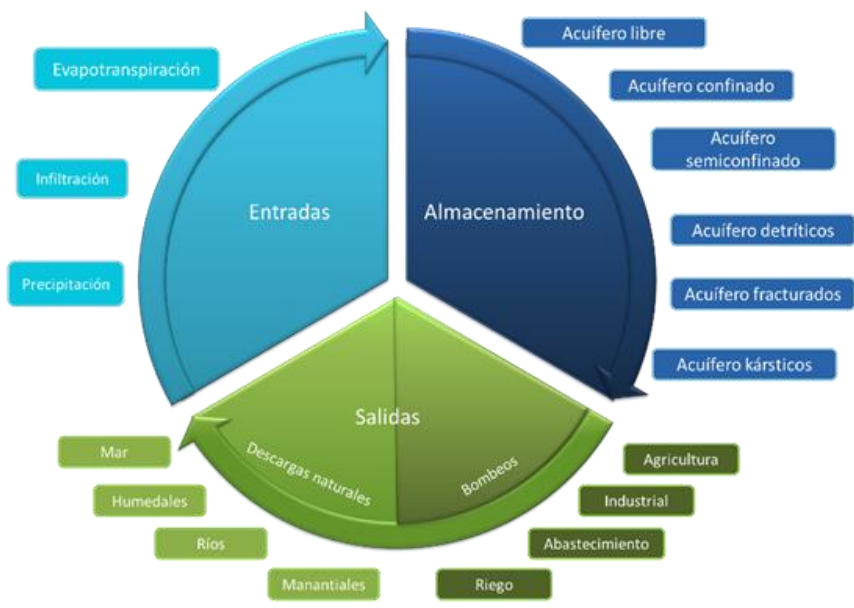

Fig.1. Fase subterránea del ciclo hidrológico. Modificado de [6].

\section{$2.1 \quad$ Entradas}

La recarga a los acuíferos dependerá fundamentalmente de la precipitación, la infiltración y la pérdida por evapotranspiración [17]. Tanto el clima como la cobertura terrestre determinan la precipitación y evapotranspiración, mientras que el suelo subyacente y la geología dictan si un excedente de agua puede ser transmitida y almacenada en el subsuelo [7].

La precipitación es el factor climático primario para la recarga de las aguas subterráneas. Tanto la magnitud de la precipitación como su intensidad, estacionalidad y frecuencia, influencian la recarga de acuíferos. Las proyecciones de los Modelos Climáticos Globales de precipitación para el siglo $\mathrm{XXI}$, indican en términos generales un patrón "rich get richer" en el que se espera que las regiones de convergencia de humedad experimenten un aumento de precipitación, y las de divergencia una disminución. Sequías más largas pueden ser alternadas con eventos de lluvias más frecuentes e intensas [7] alterando las tasas de recarga a los acuíferos, principalmente los más someros. Lluvias más fuertes podrían conducir a una mayor recarga de acuíferos, particularmente en zonas semiáridas [18], [19]. Sin embargo, el aumento de la variabilidad temporal de la precipitación puede dar lugar a que, durante los eventos de lluvia de alta intensidad, la capacidad de infiltración de los suelos pueda ser superada rápidamente, generando mayor escorrentía y por ende menor cantidad de agua que percole y recargue los acuíferos [20], [21]. Por otro lado, sequías más frecuentes y largas pueden conducir a la formación de encostramiento del suelo y suelos hidrofóbicos, de modo que durante los eventos de precipitación habrá un aumento de flujo superficial o escorrentía y una disminución en la recarga de aguas subterráneas (Fig.2). En áreas donde el acuífero es recargado a partir de los cuerpos de agua superficiales o por vías preferenciales como macroporos, es probable que lluvias con alta intensidad conduzcan a una mayor recarga [21], al igual que en zonas donde la filtración es rápida a través de la zona de raíces, por lo general en zonas semiáridas [22].
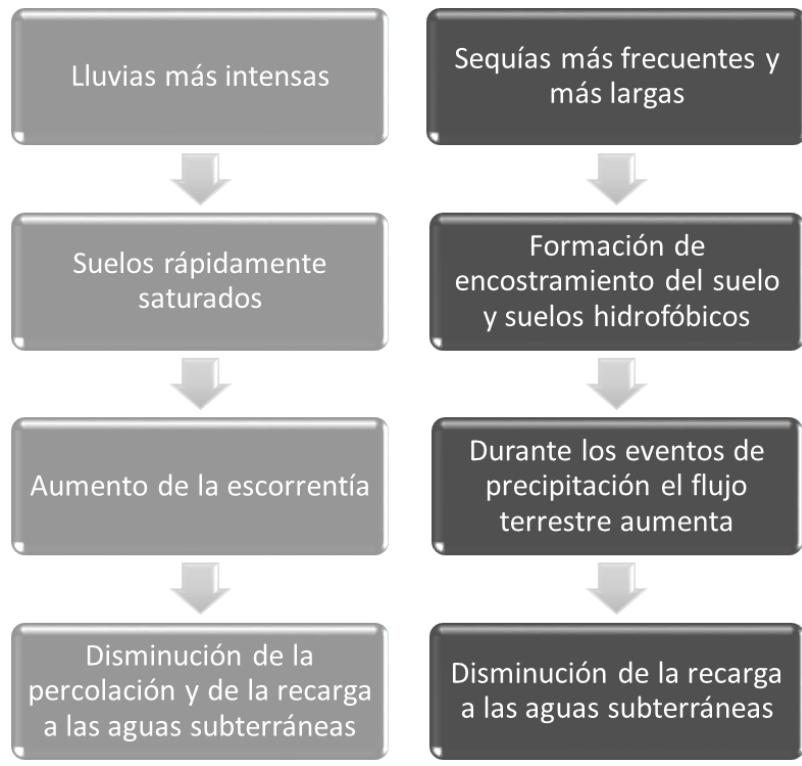

Fig.2. Disminución de la recarga a las aguas subterráneas.

La temperatura y concentraciones de $\mathrm{CO} 2$ son también importantes desde que ellos afectan la evapotranspiración y por tanto la porción de la precipitación que puede drenarse a través del perfil del suelo a los acuíferos. El aumento de la temperatura tendrá importantes consecuencias en las aguas subterráneas próximas a la superficie del terreno [23], produciéndose una disminución del agua en el acuífero que podría causar colapsos o subsidencias en el terreno y problemas geotécnicos en edificios de poblaciones urbanas. Por supuesto, las características del suelo y el tipo de cobertura vegetal, desempeñan un papel esencial en este proceso natural. El aumento en la duración y 
frecuencia de las sequías (debido al incremento de las temperaturas y variación en la precipitación) es probable que resulte en un mayor déficit de humedad del suelo. Cuando el agua del suelo se agota, la vegetación puede depender cada vez más de las aguas subterráneas para la supervivencia (si esta se almacena en las proximidades de la zona de la raíz) [15]. El aumento de CO2, sin embargo, aumentará la resistencia de las estomas de algunas plantas para retener el agua y evitar la transpiración. El cierre estomático en las plantas es un mecanismo muy eficiente para lograr su adaptación a la aridez [24]. Existe evidencia experimental que muestra que elevadas concentraciones atmosféricas de $\mathrm{CO} 2$ tienden a reducir la apertura de los estomas en las plantas, y que esto lleva a reducir las tasas de transpiración [25]-[28]; esta es una alusión que debe investigarse con mayor profundidad pues es una respuesta que varía dependiendo del tipo de plantas. En otros géneros, el incremento de $\mathrm{CO} 2$ promoverá el crecimiento y por lo tanto el aumento de la superficie de transpiración del tejido. En la actualidad existen pocos estudios sobre la respuesta de las plantas al cambio climático y como ello puede impactar en la recarga de las aguas subterráneas [11].

La posible variación del régimen de lluvias a favor de una mayor concentración de éstas, sumado al incremento de las temperaturas y de la demanda hídrica con motivo del cambio climático, modificará el proceso de infiltración del agua en el terreno y el de generación de escorrentía superficial según como se muestra en la Fig.2, además, el aumento de las extracciones generará mayor escorrentía superficial y evapotranspiración, lo que tendrá como consecuencia un descenso generalizado de los niveles freáticos en muchos acuíferos.

\subsection{Almacenamiento}

El almacenamiento es la diferencia entre la recarga y la descarga y está influenciado por las propiedades específicas de los acuíferos, tamaño y tipo. Según sea su litología o en función de la presión hidrostática, los acuíferos reaccionan de diferente forma al cambio climático.

Según su litología, los acuíferos detríticos son los más sensibles a las variaciones del nivel del agua de los ríos haciéndolos susceptibles a cualquier afección que se produzca en la cantidad y calidad de sus aguas subterráneas. Con el cambio climático la demanda hídrica en zonas próximas a los ríos y a la costa aumentará, al igual que las extracciones de agua subterránea, lo que conlleva a una disminución generalizada de los volúmenes de agua subterránea. Los acuíferos fracturados por su parte, ante aumentos en la precipitación grandes volúmenes de agua podrían ingresar dado que estos permiten una apreciable circulación de agua, sin embargo, este tipo de acuíferos tienen muy poca inercia y en época de sequía no ofrecen mucha garantía como almacén de agua subterránea. En cuanto a los acuíferos kársticos, el aumento de las emisiones de CO2 a la atmósfera podría influir en la formación de sistemas kársticos. Sin embargo, estos sistemas se podrían ver afectados ante grandes descargas de aguas subterráneas en surgencias naturales de origen kárstico, debido a la mayor concentración de las lluvias que se prevé con el cambio climático [6], [29].

En función de la presión hidrostática los acuíferos libres, también llamados no confinados o freáticos, son especialmente sensibles a las variaciones climáticas puesto que su recarga depende directamente de la infiltración de la precipitación a través del suelo, o por infiltración de agua de ríos o lagos. Por el contrario, los acuíferos confinados son poco sensibles a la variabilidad climática y los efectos del cambio climático se verían en un lapso de tiempo muy prolongado [29].

Los acuíferos más profundos reaccionan con retraso al cambio climático a gran escala, mientras que los sistemas de aguas subterráneas poco profundas son más receptivos a la variabilidad climática y al cambio global. Los impactos del cambio climático en el almacenamiento también dependerán de si es o no el agua subterránea renovable o si comprende un recurso fósil. Los sistemas acuíferos sufrirán en gran medida si la recarga mengua a su vez que las extracciones siguen en aumento debido a la demanda [30]-[32]. De hecho, según estadísticas de la FAO las extracciones mundiales de agua subterránea se han triplicado en los últimos cincuenta años, y más de la cuarta parte de las extracciones actuales no son sostenibles. Donde ocurren bajas precipitaciones la amenaza es evidente y en amplias zonas de China, India, Pakistán, Bangladesh, Irán, Estados Unidos, México y Europa se explota con mayor intensidad los acuíferos, lo que puede conllevar a una pérdida de 
reservas de agua dulce en un momento en que estos grandes almacenes juegan un papel estratégico para garantizar la seguridad hídrica y la adaptación al cambio climático, especialmente en los periodos de estiaje [33].

\section{$2.3 \quad$ Salidas}

Los impactos del cambio climático en la descarga de las aguas subterráneas son menos entendidos aún. Históricamente los estudios de agua subterránea se han centrado en la comprensión de la cantidad de agua que entra en el sistema de aguas subterráneas y si esto es adecuado para el uso humano [4]. Si estos conocimientos aún se consideran pocos, menos se ha prestado atención a los ecosistemas que las aguas subterráneas soportan, tales como la vegetación terrestre y el flujo de las aguas subterráneas a manantiales, ríos, arroyos, humedales y océanos. Si tanto la recarga como el almacenamiento de las aguas subterráneas disminuyen, también las descargas naturales de éstas disminuirán afectando por tanto a los ecosistemas dependientes. Otros sistemas afectados los comprenden aquellas comunidades, industrias y entornos que dependen de las aguas subterráneas para el abastecimiento hídrico. El cambio climático y otras presiones, pueden comprometer la disponibilidad y calidad de los recursos de agua subterránea con implicaciones significativas para la salud humana y del medio ambiente, la seguridad alimentaria y la estabilidad social y económica. Las consecuencias ambientales incluyen la reducción o eliminación de caudal base y refugios para plantas y animales acuáticos, muerte regresiva de la vegetación dependiente de aguas subterráneas y reducción del suministro de agua para los sistemas dependientes. En áreas donde se produce la salinización, por ejemplo, regiones costeras, las especies sensibles a la sal pueden desaparecer y afectar las comunidades que se abastecen del agua subterránea [6].

\subsection{Calidad del agua subterránea}

Ambas propiedades térmicas y químicas de las aguas subterráneas pueden verse afectadas por el cambio climático. La temperatura de las aguas subterráneas es un factor importante en la calidad del agua, y por lo tanto es un parámetro crucial en la gestión de la calidad de los recursos hídricos subterráneos [23]. En los acuíferos libres, la temperatura de las aguas subterráneas incrementará debido al aumento de la temperatura del aire. En zonas áridas y semiáridas, el aumento de la evapotranspiración puede conducir a una salinización de las aguas subterráneas [34]. La composición química del agua que circula a través de la zona no saturada hasta los acuíferos se puede ver afectada a causa del aumento de la temperatura, ya que se estima que entre el 6 y $7 \%$ del carbono orgánico en el suelo, se pierde por cada grado de temperatura media que aumente [6]. Por otro lado, el aumento de temperatura del agua subterránea puede tener una influencia significativa en las dinámicas ecológicas de los ecosistemas dependientes [8], [23]. Los ríos y arroyos ganadores pueden ser fuertemente influenciados por los regímenes térmicos de los acuíferos circundantes, especialmente durante la época de verano cuando el flujo base es predominante en la corriente [23].

En acuíferos costeros, es probable que el aumento del nivel del agua y tormentas en la costa, conduzcan a que haya mayor intrusión salina en los recursos hídricos subterráneos [35]-[37]. Cambios en la recarga y descarga probablemente cambiarán la vulnerabilidad de los acuíferos a la contaminación difusa [34]. En áreas donde se espera que la precipitación aumente, los contaminantes (pesticidas, materia orgánica, metales pesados, etc.) lavados del suelo a los cuerpos de agua se incrementará [38]. La recarga relacionada a eventos de fuertes lluvias también se asocia con contaminación microbiana de las aguas subterráneas poco profundas [7], [19]. Donde la recarga a los acuíferos ocurre por medio de los cuerpos de agua superficiales, la calidad del agua subterránea es más vulnerable. Donde se espera que la recarga disminuya, la calidad del agua puede también disminuir debido a una menor dilución [11], en algunos casos puede también conducir a la intrusión de agua de menor calidad de acuíferos vecinos [34]. Por otro lado, en las zonas urbanas es probable que el riesgo de contaminación sea mayor debido a una mayor densidad de población y concentración de fuentes contaminantes.

\section{CAMBIO CLIMÁTICO EN COLOMBIA}

En su Tercera Comunicación Nacional de Cambio Climático, el Instituto de Hidrología, Meteorología y 
Estudios Ambientales - IDEAM, presenta los escenarios de cambio climático de la precipitación y las temperaturas media, máxima y mínima para Colombia y sus departamentos, usando los escenarios de forzamiento radiativo impuestos por la concentración esperada de Gases de Efecto Invernadero (GEI) RCP2.6, RCP4.5, RCP6.0 y RCP8.5, definidos en el V Informe del Grupo Intergubernamental de Expertos sobre el Cambio Climático - IPCC [39]. Los escenarios presentan la posible alteración del clima (precipitación y temperatura) del futuro con respecto al clima de referencia (1976 - 2005). Estos indican que el país en su conjunto estaría afectado por el Cambio Climático, pero este cambio no será el mismo para todas las regiones del país, sino que tanto el aumento en la temperatura como el comportamiento de las precipitaciones variará para cada región del territorio nacional. Con el aumento de las emisiones globales de GEI, la temperatura media anual en Colombia podría incrementarse gradualmente en $2.14^{\circ} \mathrm{C}$ para el fin del Siglo XXI, lo que puede causar entre otras cosas, aumento en el nivel del mar, lo que comprometería a las poblaciones y ciudades asentadas en las costas; el derretimiento acelerado de los nevados y glaciares, el retroceso de páramos de los que gran parte de la población del país depende; la reducción en la productividad agropecuaria debido a mayores sequías y la mayor incidencia de fenómenos climáticos extremos. Sumado a esto, los cambios en el uso del suelo incrementarán los procesos de desertificación, pérdida de suelos productivos, pérdida de fuentes y cursos de agua, y ocasionar mayor incidencia de olas de calor especialmente en áreas urbanas [40].

Además, es muy probable que los efectos de fenómenos de variabilidad climática como el ENOS (El Niño Oscilación del sur), tengan mayor impacto en el territorio colombiano debido a un cambio gradual en la temperatura y precipitación. Así que, donde se presente el fenómeno de El Niño es probable que la disminución de lluvias y las m sequías se presenten con más fuerza, del mismo modo, en los años en que se presente el fenómeno de La Niña se esperará precipitaciones más intensas y en mayor cantidad. Las reducciones en las lluvias sumadas a los cambios en el uso del suelo pueden acelerar e intensificar los procesos de desertificación y pérdida de fuentes y cursos de agua, por el contrario, aumentos en la precipitación sumados a los cambios en el uso del suelo puede incrementar la posibilidad de deslizamientos, daño a acueductos e infraestructura vial, e inundaciones en áreas planas del país [41].

El principal cambio se proyecta en la región Andina, especialmente en las zonas de alta montaña, donde los territorios con bajas temperaturas se volverían un poco más cálidas; situación similar se esperaría en la Orinoquía y en la Sierra Nevada de Santa Marta. Las ciudades apreciarán de forma aumentada el efecto de islas de calor y los sistemas de páramos y zonas nivales en la región, se pueden ver afectados en su integridad ecológica. Los cambios más notorios de aumento de la precipitación se darían también en la región Andina (más específicamente en el eje cafetero, norte de Cauca, Nariño y sur de Norte de Santander) y disminuciones considerables en el norte del país, la Amazonía y la Orinoquía [42].

\section{EFECTOS DEL CAMBIO CLIMÁTICO SOBRE LAS AGUAS SUBTERRÁNEAS EN COLOMBIA: CASOS DE ESTUDIO}

Según lo visto anteriormente, es posible traer a colación algunas consideraciones de los impactos que tendrían los escenarios de cambio climático propuestos por el IDEAM, sobre las aguas subterráneas en el Valle de Aburrá - Antioquia [43], [44], Urabá antioqueño [45], [46] y río Ranchería en la Guajira [47], tanto en la recarga como en el almacenamiento, descarga y ecosistemas implicados. Su localización y unidades acuíferas se presentan en la Tabla 1 y Fig.3.

Tabla 1. Unidades Acuíferas del Valle de Aburrá [43], Urabá antioqueño [45] y Río Ranchería [47].

\begin{tabular}{lc}
\hline \multicolumn{1}{c}{ Unidad acuífera } & Descripción \\
\hline Acuífero Libre & $\begin{array}{c}\text { Depósitos aluviales del río } \\
\text { Medellín y sus afluentes, y } \\
\text { depósitos de vertiente } \\
\text { categorizados como flujos de lodo } \\
\text { y escombros. }\end{array}$ \\
$\begin{array}{l}\text { Acuífero } \\
\text { semiconfinado }\end{array}$ & $\begin{array}{c}\text { Depósitos de origen aluvial, } \\
\text { separados del acuífero libre por } \\
\text { una capa arcillosa, cuyo espesor } \\
\text { varía entre } 0 \text { y } 57 \text { m con una } \\
\text { media de } 12,8 \text { m. }\end{array}$ \\
Acuífero de la & Acuífero fracturado con condición \\
Dunita de Medellín & de pseudokarst en algunos \\
& sectores.
\end{tabular}




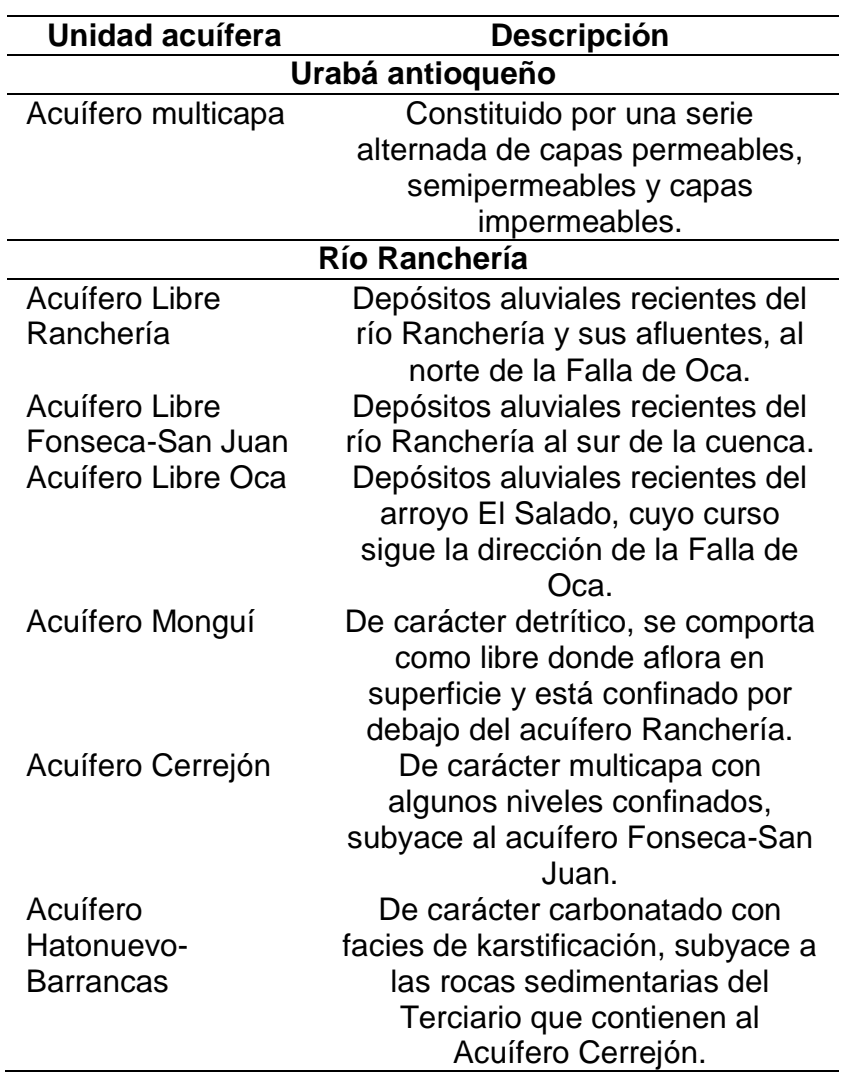

\subsection{Sistema Acuífero del Valle de Aburrá - Antioquia}

Según los escenarios de cambio climático de temperatura y precipitación propuestos por el IDEAM, se presenta un aumento de 1 a $2.2^{\circ} \mathrm{C}$ para fin de siglo, con los valores más altos en las zonas bajas del valle. La precipitación por otro lado, muestra un aumento generalizado de entre el 20 y $40 \%$ con los mayores valores en el centro del valle donde coincide con áreas urbanizadas e impermeables. Ante estos escenarios, es evidente que el Acuífero Libre es el más vulnerable al ser recargado directamente por la precipitación local, teniendo en cuenta que, según los valores de permeabilidad y transmisividad de los depósitos aluviales y de vertientes, la respuesta de esta unidad hidrogeológica a cambios meteorológicos y antrópicos es relativamente rápida. El aumento de temperatura afectaría de diferente manera las áreas de recarga, dependería en gran parte del tipo de cobertura y uso del suelo [24], [48]. En el Valle de Aburra los pastos representan el $34,61 \%$ de la totalidad de las coberturas terrestres del valle, los cultivos el $12,73 \%$, las áreas de rastrojo cubren el
$18,47 \%$, mientras que los bosques ubicados en las partes altas de la cuenca, tanto natural como plantado, ocupan aproximadamente el 19,44\% [49]. Las coberturas que mayor evapotranspiración aportarían en el Valle de Aburrá serían los bosques, rastrojos y pastos tropicales, y cultivos como el de tomate, papa, yuca, cebolla, ajo, espinaca, cilantro y café; mientras que pastos C4, cultivos de maíz, caña de azúcar y plantas CAM tales como la piña, serán más adaptables al clima cambiante. Los suelos urbanizados y desnudos o erosionados contribuirán a la mayor generación de escorrentía al no haber intercepción e infiltración de agua lluvia considerando, además, la topografía y pendientes del Valle de Aburrá.

El aumento generalizado pero concentrado de las lluvias aumentará la escorrentía superficial al superarse rápidamente la capacidad de infiltración de los suelos disminuyendo a largo plazo la entrada de agua al Acuífero Libre, aunque al principio se genere una mayor recarga. Caso diferente pasaría con la Dunita de Medellín, siendo un acuífero fracturado pseudokárstico, este aumento daría lugar a que grandes volúmenes de agua ingresen, generando una importante fuente de agua que podría llegar a ser utilizable con fines de abastecimiento, sin embargo, aún es necesaria la realización de estudios de exploración e investigación para la generación y afianzamiento de conocimiento sobre este acuífero. El acuífero semiconfinado, por el contrario, aunque no se tiene mucha información, no se vería seriamente afectado por el cambio climático, antes bien podría ser una mayor fuente de abastecimiento satisfaciendo la creciente demanda de agua y como medida de adaptación ante el cambio climático.

Los sistemas dependientes del Acuífero Libre podrían verse afectados ante una disminución de la cantidad y calidad de las aguas subterráneas con motivo de la disminución en la recarga. La cantidad de agua en los afluentes y lagos aumentará en el momento de la precipitación y generación de escorrentía, pero en periodos largos de sequía, su caudal dependerá de las salidas del agua subterránea. Los manantiales permanentes pasarían a ser estacionales afectando el suministro de agua potable a poblaciones dependientes, como ocurre generalmente en áreas rurales del Valle de Aburrá. Los demás cuerpos de aguas superficiales dependientes podrían llegar a desaparecer para fin de siglo. De hecho, en la actualidad se infiere que 


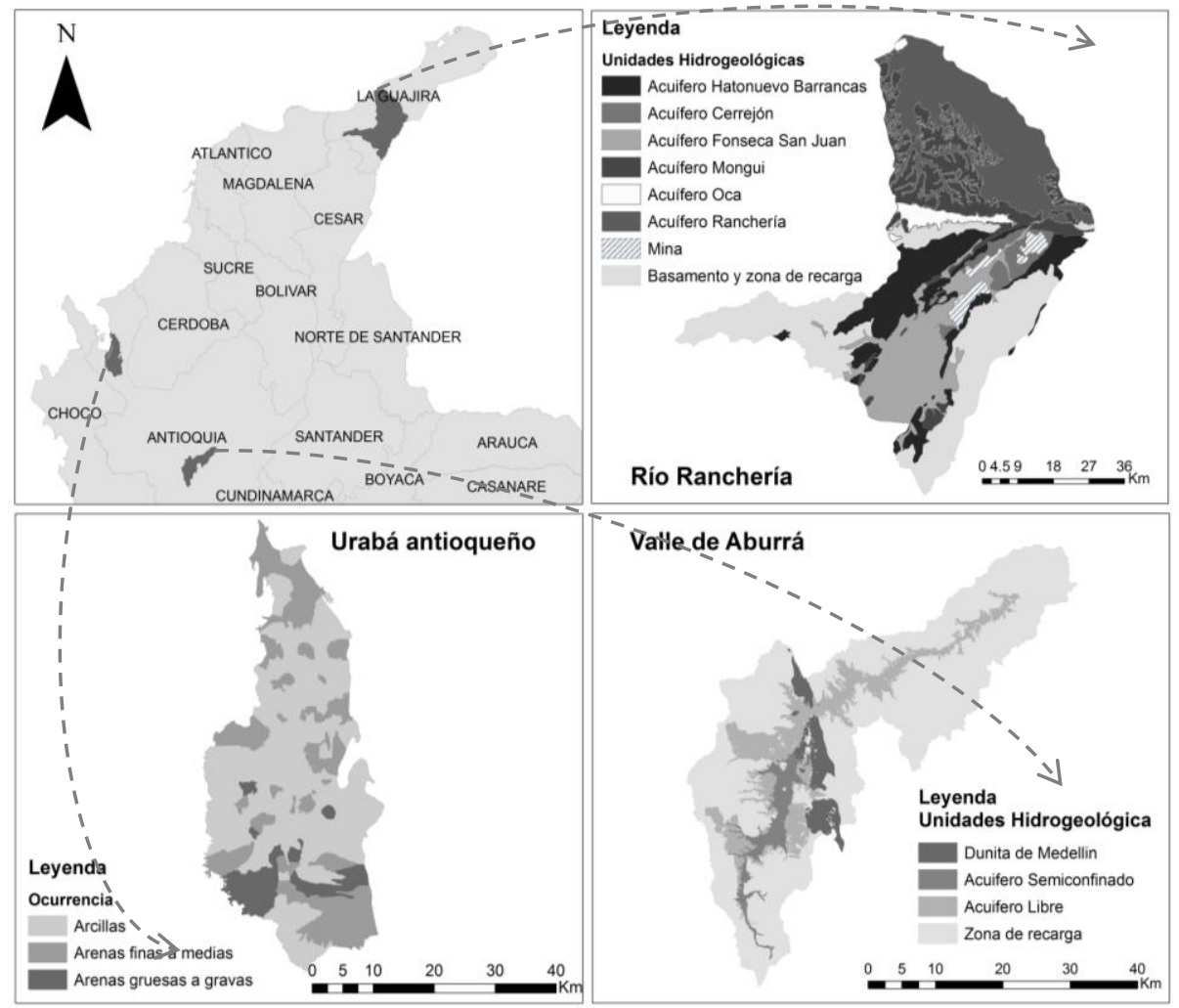

Fig.3. Localización y unidades acuíferas de casos de estudios: Valle de Aburrá [43], Urabá antioqueño [45] y Río Ranchería [47].

el nivel de los lagos del Jardín Botánico y Parque Norte del municipio de Medellín, ha estado descendiendo debido a que no les está llegando suficiente agua subterránea, esto porque en los últimos años, el aumento de construcciones e infraestructuras ha dado lugar a que sea necesario el bombeo continuo de agua para evitar la inundación de sótanos, interfiriendo así en el flujo de agua subterránea hacia los lagos.

En cuanto a la calidad, nuevamente el Acuífero Libre es el más vulnerable, generalmente la mayor contribución siendo un acuífero urbano es por contaminación antrópica. En periodos prolongados de no lluvia, la calidad del agua disminuiría debido a una menor dilución de los contaminantes, y cuando se presenten los eventos de precipitación, los contaminantes como pesticidas y materia orgánica lavados del suelo a los cuerpos de agua puede aumentar.

\subsection{Sistema Hidrogeológico del Urabá antioqueño}

Según las proyecciones del IDEAM, para final de siglo se tendrán aumentos considerables de Temperatura de entre 2,61 a $2,7^{\circ} \mathrm{C}$ en toda el área del sistema hidrogeológico, y se prevé un aumento de precipitación de un 10 a un $20 \%$. Este aumento de temperatura es de las más altas en Antioquia y repercutirá en una posible reducción de las entradas de agua al sistema. La recarga al Sistema Hidrogeológico de Urabá se estaría dando principalmente, a partir de los excedentes de precipitación que se infiltra a través de las zonas con mayor permeabilidad, zonas donde afloran materiales gruesos (arenas y gravas) respecto a los finos (arcilla) cuyo tránsito es más lento. En los niveles más profundos se deben estar dando flujos regionales que involucran agua almacenada en el subsuelo por largos períodos y que seguramente han recorrido grandes distancias. Bajo este supuesto, es posible que se esté dando una recarga indirecta desde la Serranía hacia las partes más profundas del sistema. En el estudio realizado 
por la Universidad de Antioquia y CORPOURABA [46], se observa la respuesta del sistema al régimen de explotación que se estaría dando durante la época de verano, cuando se generaría mayor uso del agua subterránea para el riego de cultivos y para diferentes procesos industriales y domésticos. La demanda de agua subterránea durante estas épocas, podrían aumentar aún más ante el escenario propuesto por el IDEAM, específicamente para la temperatura, presentándose mayores descensos en el nivel freático en el futuro.

La respuesta rápida ante eventos climáticos de este sistema [45], sumado a la necesidad económica de sobreexplotar el recurso en épocas de estiaje, hacen este acuífero vulnerable a los cambios climáticos y ambientales. Considerando que el aumento en las precipitaciones es bajo, un incremento de temperatura de más de $2^{\circ} \mathrm{C}$ aumentará aún más las tasas de evaporación y evapotranspiración. Para la zona de estudio, las coberturas que predominan son los cultivos permanentes: herbáceos (vías fotosintéticas C3 y C4), arbóreos (C3) y agroforestales; pastos limpios y mosaicos de pastos (C3 y $\mathrm{C} 4)$ y cultivos siendo los de plátano y banano los principales (C3) [46]. En este sentido, las coberturas con plantas tipo C4 poseen un uso eficiente del agua mucho mayor que las de tipo C3 [24], las de este último, que corresponden a la mayor parte de la cobertura vegetal, evapotranspiran más disminuyendo la cantidad de agua que percole hacia el acuífero y por tanto la recarga.

El sistema acuífero del Urabá mantiene una conexión importante con las fuentes superficiales de agua. Tiene asociados ecosistemas mangláricos y humedales de importancia regional y global. Las aguas subterráneas allí, sostienen varios de estos ecosistemas además de aportar al caudal base de ríos y afluentes. Según la modelación de niveles piezométricos, se observan áreas de inundación correspondientes con zonas definidas como humedales [50], [51]. En algunos sectores podría ocurrir lo contrario, en el que parte de los afluentes y humedales aportan a la recarga del sistema acuífero. Una disminución a la recarga sumado a una sobreexplotación del recurso, afectaría de gran manera estos ecosistemas dependientes, volviendo humedales y cauces permanentes en temporales y afectando su dinámica ecosistémica.
La calidad del agua subterránea en esta región costera, se ve comprometida en tanto aumenten los niveles del mar a causa del cambio climático, lo que favorecerá la mayor intrusión salina en las proximidades del Golfo.

\subsection{Sistema Acuífero del río Ranchería - Guajira}

Según proyecciones del IDEAM, se prevén aumentos significativos de temperatura para todo el departamento de La Guajira de 2,51 a $2,7^{\circ} \mathrm{C}$, y reducciones de precipitación de hasta un $20 \%$ en promedio para fin de siglo; en particular para la cuenca del Río Ranchería las mayores disminuciones de entre 30 y $40 \%$ respecto al valor actual se presentarían en Riohacha hacia la parte baja de la cuenca [40]. Este escenario para fin de siglo podría resultar desalentador aún en el ámbito hidrogeológico. Aunque las características de las unidades hidrogeológicas y de los suelos favorecen la infiltración y percolación incorporando a los acuíferos agua por recarga directa o lateral, las condiciones climatológicas imperantes en la cuenca condicionan la magnitud y distribución espacial de la recarga, al verse reducidos enormemente los excedentes hídricos que podrían incorporarse a los acuíferos [47]. Mayores temperaturas y menores precipitaciones afectarían aún más la cantidad de agua que recarga los acuíferos en la zona, especialmente los acuíferos libres, esto es las unidades hidrogeológicas Ranchería, Fonseca San Juan y Oca, cuya recarga es directa y son alimentados por cuerpos de agua superficial perennes e intermitentes (y que serían los primeros en disminuir bajo este escenario futuro).

La recarga directa al acuífero de Fonseca-San Juan se reduciría significativamente, pero seguiría recibiendo aportes lateralmente, al igual que El acuífero Cerrejón, desde las vertientes de la Sierra Nevada de Santa Marta y la Serranía del Perijá, dado que por su afectación estructural transmitirían el agua que se infiltra a ellas; además el aumento en la temperatura aceleraría el deshielo de la Sierra Nevada favoreciendo la recarga desde ésta. Respecto al acuífero de Monguí, su recarga por precipitación disminuiría en las partes donde aflora en superficie, mientras que en las partes donde se comporta como confinado, su afectación sería menor y recibiría eventualmente aportes verticales desde las unidades Oca y Ranchería. Finalmente, a través de las áreas de afloramiento de las rocas del 
cretáceo y desde las vertientes montañosas, se debe estar produciendo la recarga de la unidad hidrogeológica Hatonuevo-Barrancas, al ser un acuífero confinado no tendría impactos significativos ante un cambio climático, sin embargo, su vulnerabilidad se asocia a la actividad de minería extractiva de El Cerrejón que podría provocar su desconfinamiento [47].

Es de esperar, que la demanda del recurso hídrico subterráneo se incremente paulatinamente conforme aumente la temperatura y disminuya la precipitación; los usos actuales del agua subterránea en la cuenca corresponden a un 74,5\% en el sector doméstico, $4,85 \%$ en el sector agrícola, $6,97 \%$ a captaciones que usan el recurso en actividades hoteleras y hospitalarias, 13,28 \% para uso pecuario y en la industria $0,4 \%$ [47]. Este aumento de demanda sumado a una disminución en la recarga, presentará descensos en los almacenamientos de los acuíferos libres, por lo que los acuíferos con carácter confinado serán aún más atractivos como medio para la adaptabilidad al Cambio Climático, siempre se tengan buenas prácticas de gestión del recurso.

En la cuenca del Río Ranchería, hacia la región sur-occidental (delimitada por la Sierra Nevada de Santa Marta), las características de cobertura vegetal llevan a una mayor dominancia de la evapotranspiración que en las otras zonas de la cuenca, e influye en las condiciones del suelo aumentando los límites de almacenamiento y disminuyendo el agua disponible para la recarga. Ante el escenario propuesto por el IDEAM para fin de siglo, la vegetación presente en esta área podría depender cada vez más del agua en el subsuelo, limitando aún más la cantidad de agua que percole hacia los acuíferos. Si las sequías se vuelven severas a tal extremo que se presente mortandad de árboles y demás vegetación, se podría presentar encostramiento del suelo y suelos hidrofóbicos, dando lugar a que, durante los eventos de precipitación se genere mayor escorrentía y poca infiltración (Fig. 2).

Por otra parte, siendo el sistema hidrogeológico de la cuenca del Río Ranchería costero, la elevación del mar podría reducir el espesor del agua dulce en los acuíferos y acentuar la intrusión salina. Además, el aumento futuro de las cargas de sal causará la salinización de las aguas superficiales y aguas subterráneas poco profundas y pondrá bajo presión los volúmenes totales de las aguas subterráneas para el abastecimiento de agua potable, los usos agrícolas, la industria y los ecosistemas [35], [38].

\section{CONCLUSIONES}

Las aguas subterráneas generan un importante potencial de adaptabilidad de las poblaciones al cambio climático, debido a que son menos sensibles que las superficiales a las variaciones que este pueda producir [52]. Sin embargo, es necesaria una debida gestión y anticipación de los efectos del cambio climático que podrían poner en peligro la cantidad y calidad del recurso. En este sentido, en todo el mundo ya se han utilizado diferentes modelos para intuir en los efectos climáticos sobre los acuíferos (p. ej. [35], [53]-[62]), sin embargo, es necesario tener en cuenta al momento de cuantificar estos impactos, la incertidumbre asociada a las proyecciones climáticas (en especial de la precipitación) y a la influencia relativa de otros factores, como la respuesta de la vegetación al cambio de la temperatura y la concentración de dióxido de carbono [11], [60]. Generalmente la cuantificación de estos impactos se centra en el cambio de la precipitación y temperatura suponiendo constante otros parámetros. Los estudios de efectos climáticos indirectos, como el cambio de uso del suelo, la cubierta vegetal y las propiedades del suelo son muy pocos, y la variabilidad climática natural es a menudo ignorada. Es necesario, por tanto, que futuros estudios e investigaciones aborden los impactos indirectos climáticos y no climáticos para la obtención de predicciones más realistas de la respuesta hidrogeológica al clima futuro, y así, poder plantear estrategias de adaptación con las aguas subterráneas de una manera sostenible [4], [9], [52], [63]-[65].

Por otro lado, es necesario tener presente que no solo el cambio climático tendrá efectos adversos sobre la cantidad y calidad de los recursos hídricos subterráneos, en muchas áreas estos serán eclipsados por impactos no climáticos que constituyen amenazas latentes incluyendo el crecimiento demográfico, la demanda de alimentos y por ende de la actividad agropecuaria, cambios en el uso del suelo, urbanización y factores socioeconómicos que influyen en la capacidad de gestionar adecuadamente los recursos de agua subterránea [4], [5]. 
A partir de unos elementos conceptuales, teniendo en cuenta las características hidrogeológicas de tres sistemas acuíferos de Colombia y considerando las proyecciones del IDEAM sobre los posibles impactos del cambio climático en el país, se elaboró una descripción cualitativa acerca de los impactos que ello podría representar sobre las aguas subterráneas. Sera mediante ejercicios de monitoreo, reconstrucción de información y modelación a partir de series históricas que se puedan efectuar simulaciones y pronósticos que ofrezcan escenarios probables para argumentar medidas tendientes a una mejor gestión.

\section{AGRADECIMIENTOS}

A COLCIENCIAS patrimonio autónomo del Fondo Nacional de Financiamiento para la Ciencia, la Tecnología y la Innovación Francisco José de Caldas, por su apoyo en el convenio 111571450898.

\section{REFERENCIAS BIBLIOGRÁFICAS}

[1] Shimanko, I. Comprenhensive assessment of the freshwater resources of the world. Stockholm: Stockholm Environment Institute: Stockholm: Karim Hultcrantz., 1997.

[2] WWAP. The United Nations World Water Development Report 3: Water in a Changing World, World Water Assessment Programme. UNESCO Publishing, 2009.

[3] Döll, P., Hoffmann-Dobrev, H., Portmann, F.T., et al. Impact of water withdrawals from groundwater and surface water on continental water storage variations, J. Geodyn., 59-60, 143-156, 2012.

[4] Clifton, C., Evans, R., Hayes, S., et al. Water and climate change: impacts on groundwater resources and adaptation options, Water Working Notes, 2010.

[5] Treidel, H., Martin-Bordes, J.L. y Gurdak, J.J. Climate Change Effects on Groundwater Resources: A Global Synthesis of Findings and Recommendations, CRC Press, London, UK, 2011.

[6] Pernía Llera, J.M. y Fornés Azcoiti, J.M. Cambio Climático y Agua Subterránea, Enseñanza las Ciencias la Tierra, 17(2), 172-178, 2009.

[7] Taylor, R.G., Todd, M.C., Kongola, L., et al. Evidence of the dependence of groundwater resources on extreme rainfall in East Africa, Nat. Clim. Chang., 3(4), 374-378, 2013.
[8] Kløve, B., Ala-Aho, P., Bertrand, G., et al. Climate change impacts on groundwater and dependent ecosystems, J. Hydrol., 518, 250-266, 2014.

[9] Green, T.R., Taniguchi, M., Kooi, H., et al. Beneath the surface of global change: Impacts of climate change on groundwater, J. Hydrol., 405(34), 532-560, 2011.

[10] Kundzewicz, Z.W. y Döll, P. Will groundwater ease freshwater stress under climate change? Hydrol. Sci. J., 54(4), 665-675, 2009.

[11] Kundzewicz, Z.W., Mata, L.J., Arnell, N.W., et al. Freshwater resources and their management. En: Freshwater resources and their management. Climate Change 2007: Impacts, Adaptation and Vulnerability. Contribution of Working Group II to the Fourth Assessment Report of the Intergover nmental Panel on Climate Change (Eds. M.L. Parry, O.F. Canziani, J.P. Palutikof, et al.). Cambridge, UK, 173-210, 2007.

[12] Jiménez, B.E., Oki, T., Arnell, N.W., et al. Freshwater resources. En: Climate Change 2014: Impacts, Adaptation, and Vulnerability. Part A: Global and Sectoral Aspects. Contribution of Working Group II to the Fifth Assessment Report of the Intergovernmental Panel on Climate Change (Eds. C.B. Field, V.R. Barros, D.J. Dokken, et al.). Cambridge University Press, Cambridge, United Kingdom and New York, NY, USA, 229-269, 2014.

[13] Hernández, M.A. Aguas subterráneas y cambios climáticos. Disponible en: http://sedici.unlp.edu.ar/bitstream/handle/10915/269 56/Documento_completo.pdf?sequence $=1$ [Consultado el 03 de marzo de 2015].

[14] Panwar, S. y Chakrapani, G.J. Climate Change and Its Impact on Groundwater Resources, Curr. Sci., 105(1), 37-46, 2013.

[15] Dragoni, W. y Sukhija, B.S. Climate change and groundwater: a short review. En Climate Change and Groundwater Geological Society, London, Special Publications, 288(1), 1-12, 2008.

[16] Kumar, C.P. Climate Change and Its Impact on Groundwater Resources Int. J. Eng. Sci., 1(5), 4360, 2012.

[17] Döll P. y Fiedler, K. Global-scale modeling of groundwater recharge, Hydrol. Earth Syst. Sci. Discuss., 12(3), 863-885, 2008.

[18] Owor, M., Taylor, R.G., Tindimugaya, C. y Mwesigwa, D. Rainfall intensity and groundwater recharge: empirical evidence from the Upper Nile Basin, Environ. Res. Lett., 4(3), 35009(6), 2009.

[19] Taylor, R.G., Scanlon, B., Döll, P., et al. Ground water and climate change, Nat. Clim. Chang., 3(4), 322-329, 2013. 
[20] International Association of Hydrogeologists and British Geological Survey, Strategic Overview Series: Global change and groundwater. Disponible en: https://iah.org/wp-content/uploads/2016/07/IAHGlobal-Change-Groundwater-14-June-2016.pdf [Consultado el 12 de agosto de 2016].

[21] Döll P. y Flörke, M. Global-scale estimation of diffuse groundwater recharge, Systems Research, 2005.

[22] Liu, H.-H. Impact of climate change on groundwater recharge in dry areas: An ecohydrology approach, J. Hydrol., 407(1-4), 175183, 2011.

[23] Menberg, K., Blum, P., Kurylyk, B.L. y Bayer, P. Observed groundwater temperature response to recent climate change, Hydrol. Earth Syst. Sci., 18(11), 4453-4466, 2014.

[24] González Medrano, F. Adaptaciones de las plantas a la aridez. En: Las zonas áridas y semiáridas de México y su vegetación (Ed. F. González Medrano), México, D.F, 53-84, 2012.

[25] Mengis, N., Keller, D.P., Eby, M. y Oschlies, A. Uncertainty in the response of transpiration to $\mathrm{CO}_{2}$ and implications for climate change, Environ. Res. Lett., 10, 094001, 2015.

[26] Keenan, T.F., Hollinger, D.Y., Bohrer, G., et al., Increase in forest water-use efficiency as atmospheric carbon dioxide concentrations rise, Nature, 499, 324-327, 2013.

[27] Kruijt, B., Witte, J., Jacobs, C. y Kroon, T. Effects of rising atmospheric $\mathrm{CO}_{2}$ on evapotranspiration and soil moisture: a practical approach for the Netherlands, J. Hydrol., 349(3-4), 257-267, 2008.

[28] Bethenod, O., Ruget, F., Katerji, N., Combe, L. y Renard, D. Impact of atmospheric $\mathrm{CO}_{2}$ concentration on water use efficiency of maize, Maydica, 46(2), 75-80, 2001.

[29] López-Geta, J.A., Fornés, J.M., Ramos, G. y Villarroya, F. Las aguas subterráneas: Un recurso natural del subsuelo, 4th ed. Madrid: Instituto Geológico y Minero de España, y Fundación Marcelino Botín, 2009.

[30] Sapriza-Azuri, G., Jódar, J., Carrera, J. y Gupta, H.V. Toward a comprehensive assessment of the combined impacts of climate change and groundwater pumping on catchment dynamics, J. Hydrol., 529, 1701-1712, 2015.

[31] Zaveri, E., Grogan, D., Fisher-Vanden, K., et al. Invisible water, visible impact: groundwater use and Indian agriculture under climate change, Environ. Res. Lett., 11, 084005, 2016.

[32] Meixner, T., Manning, A., Stonestrom, D., et al. Implications of projected climate change for groundwater recharge in the western United States, J. Hydrol., 534, 124-138, 2016.

[33] FAO, Llamamiento urgente para evitar el agotamiento irreversible de las aguas subterráneas, $2015 . \quad$ Disponible en: http://www.fao.org/news/story/es/item/283110/icode 1. [Consultado el 21 de mayo de 2016].

[34] Van Vliet, M. Impact of climate change on groundwater review. IGRAC report for TNO Bouw en Ondergrond, 2007.

[35] Oude Essink, G.H.P., van Baaren, E.S. y de Louw, P.G.B. Effects of climate change on coastal groundwater systems: A modeling study in the Netherlands, Water Resour. Res., 46, W00F04, 2010.

[36] Werner, A.D., Ward, J.D., Morgan, L.K., Simmons, C.T., Robinson, N.I. y Teubner, M.D. Vulnerability Indicators of Sea Water Intrusion, Ground Water, 50(1), 48-58, 2012.

[37] Werner, A.D., Bakker, M., Post, V., et al. Seawater intrusion processes, investigation and management: Recent advances and future challenges, Adv. Water Resour., 51, 3-26, 2013.

[38] Intergovernmental Panel on Climate Change IPCC, Climate Change 2007: The Physical Science Basis. Contribution of Working Group I to the Fourth Assessment Report of the Intergovernmental Panel on Climate Change. Cambridge University Press, 2007.

[39] Intergovernmental Panel on Climate Change IPCC, Climate Change 2014: Synthesis Report. Contribution of Working Groups I, II and III to the Fifth Assessment Report of the Intergovernmental Panel on Climate Change. Geneva, Switzerland, 2014.

[40] IDEAM, PNUD, MADS, DNP, and CANCILLERÍA. Nuevos Escenarios de Cambio Climático para Colombia 2011-2100 Herramientas Científicas para la Toma de Decisiones - Enfoque Nacional - Departamental: Tercera Comunicación Nacional de Cambio Climático. Bogotá D. C., 2015.

[41] IDEAM, PNUD, MADS, DNP, and CANCILLERÍA. Nuevos Escenarios de Cambio Climático para Colombia 2011-2100 Herramientas Científicas para la Toma de Decisiones - Enfoque Nacional - Regional: Tercera Comunicación Nacional de Cambio Climático. Bogotá D. C., 2015. [42] Armenta, G.E., Dorado, J., Rodríguez, A.O. y Ruiz, J.F. Escenarios de Cambio Climático para Precipitación y Temperaturas en Colombia, IDEAM $2014 . \quad$ Disponible en: http://modelos.ideam.gov.co/media/dynamic/escena rios/escenarios-rcp-2014.pdf [Consultado el 13 de febrero de 2015] 
[43] AMVA y Universidad de Antioquia. Determinación y Protección de las Potenciales Zonas de Recarga en el Centro y Sur del Valle de Aburrá. Informe final. Contrato 254 de 2013., Medellín, 2013.

[44] Betancur, T., Martínez, C., García, E. y Escobar, J. Identification and characterization of regional water flows contributing to the recharge of an unconfined aquifer, Revista Facultad de Ingeniería, 85, 70-85, 2017.

[45] Universidad de Antioquia y CORPOURABA. Plan de Manejo Ambiental de Acuífero Sistema Hidrogeológico Golfo de Urabá. Medellín, 2016.

[46] Universidad de Antioquia y CORPOURABA. Convenio Interadministrativo de Cooperación 136 de 2013. Medellín, 2014.

[47] MADS, CORPOGUAJIRA, y Universidad de Antioquia. Plan de Manejo Ambiental de AcuíferoPMAA Cuenca del Río Ranchería. Convenio Interadministrativo 143 de 2013. Medellín, 2013.

[48] Campbell, N.A. y Reece, J.B. Biología. Médica Panamericana, 2007.

[49] Universidad Nacional. Plan de Ordenación y Manejo de la cuenca del río Aburrá - POMCA. Medellín, 2007

[50] CORPOURABA y Universidad de Medellín. Consultoría para delimitar los humedales asociados al río León en el área de influencia de los Municipios de Apartadó, Carepa, Chigorodó y Turbo, a través de técnicas que permitan modelar la función, estructura y composición de los humedales y evaluar la magnitud del daño generado por las modificaciones en el paisaje y en el cambio de uso del suelo a una escala 1:25.000. Medellín, 2012.

[51] Instituto Alexander von Humboldt y Universidad de Antioquia. Identificación y caracterización de sistemas de flujo entre humedales y aguas subterráneas. Medellín, 2015.

[52] Evans, R., Clifton, C., Hayes, S. y Hirji, R. Adaptation Options for the Impact of Climate Change on Groundwater. Water Working Notes, World Bank, 25, 2010.

[53] Portmann, F.T., Döll, P., Eisner, S. y Flörke, M. Impact of climate change on renewable groundwater resources: assessing the benefits of avoided greenhouse gas emissions using selected CMIP5 climate projections, Environ. Res. Lett., 8, 024023, 2013.

[54] Crosbie, R.S., McCallum, J.L., Walker, G.R. y Chiew, F.H.S. Modelling climate-change impacts on groundwater recharge in the Murray-Darling Basin, Australia, Hydrogeol. J., 18(7), 1639-1656, 2010.
[55] Crosbie, R.S., Pickett, T., Mpelasoka, F.S., Hodgson, G., Charles, S.P. y Barron, O.V. An assessment of the climate change impacts on groundwater recharge at a continental scale using a probabilistic approach with an ensemble of GCMs, Clim. Change, 117(1-2), 41-53, 2013.

[56] Goderniaux, P., Brouyère, S., Fowler, H.J., et al. Large scale surface-subsurface hydrological model to assess climate change impacts on groundwater reserves, J. Hydrol., 373(1-2), 122138, 2009

[57] Ertürk, A., Ekdal, A., Gürel, M., Karakaya, N., Guzel, C. y Gönenç, E. Evaluating the impact of climate change on groundwater resources in a small Mediterranean watershed, Sci. Total Environ., 499, 437-447, 2014.

[58] Awan, U.K. y Ismaeel, A. A new technique to map groundwater recharge in irrigated areas using a SWAT model under changing climate, J. Hydrol., 519, 1368-1382, 2014.

[59] Raposo, J.R., Dafonte, J. y Molinero, J. Assessing the impact of future climate change on groundwater recharge in Galicia-Costa, Spain, Hydrogeol. J., 21(2), 459-479, 2012.

[60] Kingston, D.G. y Taylor, R.G. Sources of uncertainty in climate change impacts on river discharge and groundwater in a headwater catchment of the Upper Nile Basin, Uganda, Hydrol. Earth Syst. Sci., 14(7), 1297-1308, 2010.

[61] Jackson, C.R., Bloomfield, J.P. y Mackay, J.D. Evidence for changes in historic and future groundwater levels in the UK, Prog. Phys. Geogr., 39, 49-67, 2015.

[62] Goderniaux, P., Brouyère, S., Blenkinsop, S., et al. Modeling climate change impacts on groundwater resources using transient stochastic climatic scenarios, Water Resour. Res., 47, W12516, 2011.

[63] Pasini, S., Torresan, S., Rizzi, J., Zabeo, A., Critto A. Marcomini, A. Climate change impact assessment in Veneto and Friuli Plain groundwater. Part II: a spatially resolved regional risk assessment, Sci. Total Environ., 440, 219-35, 2012.

[64] Holman, I.P., Allen, D.M., Cuthbert, M.O. y Goderniaux, P. Towards best practice for assessing the impacts of climate change on groundwater, Hydrogeol. J., 20, 1-4, 2012.

[65] Sekhar, M., Shindekar, M., Tomer, S.K. y Goswami, P. Modeling the vulnerability of an urban groundwater system due to the combined impacts of climate change and management scenarios, Earth Interact., 17(10), 1-25, 2013. 